Pacific

Journal of

Mathematics

\title{
CYCLIC PROPERTIES OF VOLTERRA OPERATOR
}

Fernando León-SaAvedra and Antonio Piqueras-Lerena 


\title{
CYCLIC PROPERTIES OF VOLTERRA OPERATOR
}

\author{
Fernando León-SaAvedra and Antonio Piqueras-Lerena
}

\begin{abstract}
A bounded linear operator $T$ defined on a Hilbert space $H$ is said to be supercyclic if there exists a vector $x \in H$ such that the set $\left\{\lambda T^{n} x: n \in \mathbb{N}, \lambda \in \mathbb{C}\right\}$ is dense in $H$. In the present work, two open questions posed by N. H. Salas and J. Zemánek respectively, are solved. Namely, we will exhibit that the classical Volterra operator $V$ and the identity plus Volterra operator $I+V$ are not supercyclic.
\end{abstract}

\section{Introduction.}

This paper deals with the classical Volterra operator $V$ which was introduced in 1896 . It is defined on the Hilbert space $L^{2}[0,1]$ by

$$
V f(x)=\int_{0}^{x} f(s) d s .
$$

An operator $T$ on a Hilbert space $H$ is said to be supercyclic if there exists a vector $x \in H$ such that the projective orbit $\left\{\lambda T^{n} x: \lambda \in \mathbb{C}, n \in \mathbb{N}\right\}$ is dense in $H$. The concept of supercyclicity was introduced originally in $[\mathbf{H W}]$ by Hilden and Wallen. Supercyclicity stands in the midway between hypercyclicity and cyclicity. An operator is said to be hypercyclic if there exists a vector whose orbit under $T$ is dense. On the other hand, if the linear span of some orbit is dense, the operator is called cyclic.

We have two goals:

a) To show that $V$ cannot be supercyclic on $L^{2}[0,1]$, and

b) the identity plus Volterra operator $I+V$ is not supercyclic on $L^{2}[0,1]$.

The first question was posed by N. H. Salas in [Sa] the second one by J. Zemánek in personal communication. In Section 2 we will renew acquaintance with the Volterra operator by proving that $V$ and $I+V$ are not hypercyclic, however they are cyclic. Section 3 is devoted to prove our main result.

Volterra operator has been studied by several authors. The norm of Volterra operator is $2 / \pi$ (see [Ha, Problem 149]). The problem's book of P. R. Halmos contains several nice results (some of them not so elementary) related with Volterra operator. The asymptotic behaviour of the norm $\left\|V^{n}\right\|$ is described in $[\mathbf{L R}]$. The most interesting fact about the Volterra operator is the determination of its invariant subspace lattice (see [Co, Chapter 4], 
and $[\mathbf{B r}],[\mathbf{D i x}],[\mathbf{D o n}],[\mathbf{K a}]$ and $[\mathbf{S a r}])$. Although Volterra operator is more than a hundred years old however still there exist several open questions, for example, it is not known the exact norm $\left\|V^{n}\right\|$ (see $[\mathbf{L R}]$ ); in [Ts] appear new results about Volterra operator.

\section{Hypercyclicity and cyclicity. Elementary facts.}

The Volterra operator is quasinilpotent. Thus the orbit of every vector converges to zero. Therefore $V$ cannot be hypercyclic.

For the identity plus Volterra case the argument is not so easy. The following result was pointed to the authors by J. Zemánek:

Proposition 2.1. Identity plus Volterra operator is not Hypercyclic on $L^{2}[0,1]$.

Proof. The proof is based in this fact: The inverse of $(I+V)$ is power bounded (see [Ha, Problem 150]). Thus the orbit of any vector under $(I+$ $V)^{-1}$ is bounded, therefore $(I+V)^{-1}$ cannot be hypercyclic. The result follows from a result of Herrero and Kitai which asserts that an invertible operator is hypercyclic if and only if its inverse is hypercyclic (see $[\mathbf{H K}]$ ).

However both operators are cyclic. Basically this fact is consequence of Weierstrass's Theorem.

Proposition 2.2. Volterra and identity plus Volterra operators are cyclic.

Proof. Let us denote by $L_{\mathbb{R}}^{2}[0,1]$ the subspace $\left\{f \in L^{2}[0,1]\right.$ : such that $f[0,1] \subset \mathbb{R}\}$. The orbit of the identity function 1 under $V$ is the set

$$
\operatorname{Orb}(V, 1)=\left\{1, x, \frac{x^{2}}{2}, \ldots, \frac{x^{n}}{n !}, \ldots\right\} .
$$

By Weierstrass's Theorem, the linear span of $\operatorname{Orb}(V, 1)$ is dense in $L_{\mathbb{R}}^{2}[0,1]$. That is, $V$ is cyclic on $L_{\mathbb{R}}^{2}[0,1]$. Pick $f \in L^{2}[0,1]$ and $\varepsilon>0$. The function $f=u+i v$ with $u, v \in L_{\mathbb{R}}^{2}[0,1]$, therefore there exists polynomials $p_{u}, p_{v}$ such that $\left\|p_{u}(V) 1-u\right\|^{2}<\varepsilon / 2$ and $\left\|p_{v}(V) 1-v\right\|^{2}<\varepsilon$. Thus

$$
p_{u}(x)=u_{0}+u_{1} x+\cdots+u_{n} x^{m} \quad p_{v}(x)=v_{0}+v_{1} x+\cdots+v_{m} x^{m}
$$

with $u_{i}, v_{i} \in \mathbb{R}$, let us consider $p(z)=\sum_{k=0}^{m} a_{k} z^{k}$ with $a_{k}=u_{k}+i v_{k}$, $k=0, \ldots, m$, and compute

$$
\begin{aligned}
\|f-p(V)(1)\|^{2} & =\left\|u+i v-p_{u}(V)(1)-i p_{v}(V)(1)\right\|^{2} \\
& =\left\|u-p_{u}(V)(1)\right\|^{2}+\left\|v-p_{v}(V)(1)\right\|^{2}<\varepsilon,
\end{aligned}
$$

therefore 1 is a cyclic vector for $V$. For the case of $I+V$ the proof is similar. 


\section{3. (Non) Supercyclicity.}

The adjoint of Volterra operator is defined by

$$
V^{\star} f(x)=\int_{x}^{1} f(s) d s
$$

that is, it is an integral operator. It easy to compute that $\sigma_{p}\left(V^{\star}\right)=\emptyset$. Observe that Volterra operator is defined on complex valued functions. The following result which appear in $[\mathbf{L M}]$ will reduce our problem to real functions.

Theorem 3.1 (Positive-Supercyclicity's Theorem). Let $T$ be a bounded linear operator defined on a separable Banach space $\mathcal{B}$. If $\sigma_{p}\left(T^{\star}\right)=\emptyset$ then $T$ is supercyclic if and only if there exists a vector $x \in \mathcal{B}$ such that $\left\{r T^{n} x\right.$ : $r>0, n \in \mathbb{N}\}$ is dense in $\mathcal{B}$.

Theorem 3.2. Volterra and the identity plus Volterra operators are not supercyclic on $L^{2}[0,1]$.

Proof. Let us denote by $T=V$ or $I+V$. The proof will be done in several steps:

(1) If $T$ is supercyclic on $L^{2}[0,1]$ then $T$ is supercyclic on $L_{\mathbb{R}}^{2}[0,1]$.

Proof. Let us denote by $f=u+i v$ a supercyclic vector for $T$. Observe that $T\left(L_{\mathbb{R}}^{2}[0,1]\right) \subset L_{\mathbb{R}}^{2}[0,1]$ and $T^{n} f=T^{n} u+i T^{n} v$. It is easy to see (using the positive-supercyclicity's Theorem) that the function $u$ is supercyclic for $T$ on $L_{\mathbb{R}}^{2}[0,1]$.

(2) If $f \in L_{\mathbb{R}}^{2}[0,1]$ is a continuous function (more precisely, there exists a continuous function in the coset determined by $f$ ) and $f$ is a supercyclic vector for $T$ then the point 0 is an accumulation point of zeros of $f$.

Proof. Observe that if $f$ is a continuous function so that $f$ is positive (respectively negative) on $[0, \delta]$ then the function $V f(x)$ is also positive (respectively negative) on $[0, \delta]$. Since $T f$ is a continuous function we obtain that the orbit under $T$ of $f$ is positive (negative) a.e. $[0, \delta]$. By way of contradiction suppose that $\delta \in(0,1]$ is the smaller zero of $f$ and without loss of generality suppose that $f$ is positive on $(0, \delta)$. In this situation the function -1 is separated more than $\delta$ from the set

$$
\left\{c T^{n} f: c>0, n \in \mathbb{N}\right\} .
$$

Therefore $f$ cannot be supercyclic for $T$.

(3) If $f \in L_{\mathbb{R}}^{2}[0,1]$ is a continuous function, and $f$ is a supercyclic vector for $T^{\star}$ then the point 1 is an accumulation point of zeros of $f$. 
Proof. The proof of (3) is analogous. It is sufficient to observe that if $f$ is a continuous function on $[0,1]$ and $f$ is positive on $[\delta, 1]$ with $\delta \in[0,1)$ then the orbit under $T^{\star}$ of $f$ is positive a.e. $[\delta, 1]$.

(4) The operator $T$ is supercyclic if and only if $T^{\star}$ is supercyclic.

Proof. Let us consider the isomorphism $R: L^{2}[0,1] \rightarrow L^{2}[0,1]$ defined by $R f(x)=f(1-x)$. Observe that $T=R T^{\star} R^{-1}$. Since Supercyclicity is invariant under similarity we obtain (4).

(5) Suppose that $V$ is supercyclic. Then there exists a supercyclic vector $f$ for $V$ which is so that the point 1 is an accumulation point of zeros of $V^{n} f$ for each integer $n$. Analagously, if $I+V$ is supercyclic then there exists a supercyclic vector $f$ for $(I+V)$ such that the point 1 is an accumulation point of zeros of the function $V(I+V)^{n} f$ for each integer $n$.

Proof. Let us suppose that $V$ is supercyclic, let us denote by $G$ the set of supercyclic vectors for $V$. It is well-known that the set of supercyclic vectors for a supercyclic bounded linear operator is a $\mathrm{G}-\delta$ dense subset. By (4) let us denote by $G_{\star}$ the set of supercyclic vectors for $V^{\star}$. Since $V$ is continuous the set $V^{-n}\left(G_{\star}\right)$ is also a G- $\delta$ dense subset. Therefore the intersection $H=\bigcap_{n=1}^{\infty} V^{-n}\left(G_{\star}\right) \bigcap G$ contains a dense subset. Pick $f \in H$. Clearly $f$ is supercyclic for $V$, on the other hand if $n \geq 1, V^{n} f \in G_{\star}$ and $V^{n} f$ is a continuous function. Therefore by (3) the point 1 is an accumulation point of zeros of $V^{n} f$.

For the second part let us consider the set $\bigcap_{n=1}^{\infty}(I+V)^{-n} V^{-1} G_{\star} \bigcap G$ where $G$ and $G_{\star}$ denote now the sets of supercyclic vectors for $(I+V)$ and $(I+V)^{\star}$ respectively. The rest of the proof runs as before.

(6) The Volterra and the identity plus Volterra operators are not supercyclic on $L_{\mathbb{R}}^{2}[0,1]$.

Proof. We first prove that Volterra operator is not supercyclic. It is sufficient to show that the orbit $V^{n} f$ of a possible supercyclic vector $f$ is orthogonal to the constants, that is, $\left\langle V^{n} f, 1\right\rangle=0$ for all $n$. Fix $\epsilon>0$. If $V$ is supercyclic let us consider the supercyclic function $f$ which guarantee (5). For $n \geq 1$ let us denote by $c_{n}$ a zero of $V^{n+1} f$ with $c_{n} \geq 1-\epsilon$. Since $V^{n+1} f$ is a primitive function of $V^{n} f$ by applying Barrow's formula we have:

$$
\begin{aligned}
\left|\left\langle V^{n} f, 1\right\rangle\right|^{2} & =\left(\left|\int_{0}^{c_{n}} V^{n} f(s) d s\right|+\left|\int_{c_{n}}^{1} V^{n} f(s) d s\right|\right)^{2} \\
& =\left|\int_{c_{n}}^{1} V^{n} f(s) d s\right|^{2} \\
& \leq\left(1-c_{n}\right) \int_{c_{n}}^{1}\left|V^{n} f(s)\right|^{2} d s \\
& \leq\left(1-c_{n}\right)\left\|V^{n} f\right\|^{2} \leq \varepsilon\left\|V^{n} f\right\|^{2} .
\end{aligned}
$$


Since $\epsilon>0$ is arbitrarily small (and independent of $n$ ) we obtain $\left\langle V^{n} f, 1\right\rangle=0$ for all $n$, that is $f$ is not cyclic, a contradiction. For the case of $I+V$ the proof is similar.

Thus, by (1) and (6) the proof of Theorem 3.2 is established.

Observe that although the results are stated in the space $L^{2}[0,1]$ the proofs runs as well for the spaces $L^{p}[0,1], 1 \leq p<\infty$.

When this paper was being accepted for publication, we were kindly informed by Prof. Joel H. Shapiro about the reference $[\mathbf{G M}]$ where the authors have obtained the Theorem 3.2 independently.

The authors are deeply grateful to professor Joel Shapiro since the first day that read ours humble work. We want to also thank M. Cepedello Boiso for interesting comments.

\section{References}

[Br] M.S. Brodskii, On a problem of I.M. Gelfand, Uspekhi Mat. Nak., 12 (1957), 129132, MR $20 \# 1229$.

[Co] J.B. Conway, A Course in Operator Theory, Rhode Island, Amer. Math. Soc., 2000, MR 2001d:47001, Zbl 0936.47001.

[Dix] J. Dixmier, Les operateurs permutables á l'operateur integral, Portugal Math., 8 (1949), 73-84, Zbl 0036.35703.

[Don] W.F. Donoghue, The latice of invariant subspaces of quasi-nilpotent completely continuous transformation, Pacific J. Math., 7 (1957), 1031-1035, MR 19,1066f, Zbl 0078.29504.

[GM] E. Gallardo and A. Montes, Volterra operator is not supercyclic, Integral Equations and Operator Theory, to appear.

[Ha] P.R. Halmos, A Hilbert Space Problem Book, New York, Springer Verlag, 1967, MR 34 \#8178, Zbl 0144.38704.

[HK] D.A. Herrero and C. Kitai, On invertible hypercyclic operators, Proc. Amer. Math. Soc., 116 (1992), 873-875, MR 93a:47023, Zbl 0780.47006.

[HW] H.M. Hilden and L.J. Wallen, Some cyclic and non-cyclic vectors of certain operators, Indiana Univ. Math. J., 23 (1974), 557-565, MR 484796, Zbl 0274.47004.

[Ka] G.K. Kalisch, On similarity, reducing manifolds, and unitary equivalence of certain Volterra operators, Ann. Math., 66 (1957), 481-494, MR 19,970a, Zbl 0078.09602.

[LM] F. León-Saavedra and V. Müller, Rotations of hypercyclic and supercyclic operators, preprint.

[LR] G. Little and J.B. Reade, Estimates for the norm of the n-th indefinite integral, Bull. London Math. Soc., 30 (1998), 539-542, MR 99g:47068, Zbl 0931.47032.

[Sa] N.H. Salas, Supercyclicity and weighted shifts, Studia Math., 135 (1999), 55-74, MR 2000b:47020, Zbl 0940.47005.

[Sar] D. Sarason, A remark on the Volterra operator, J. Math. Anal. Appl., 12 (1965), 244-246, MR 33 \#580, Zbl 0138.38801.

[Ts] D. Tsedenbayar, On the power boundedness of certain Volterra operator pencils, Studia Math., 156 (2003), 59-66, CMP 1961061. 
Received August 12, 2002. This work was supported partially by the Grants FQM- 257 and "Vicerrectorado de investigación de U.C.A".

Departamento de Matemáticas

UNIVERSIDAD DE CÁDIZ

C/ SACRAmento 82, 11003-CÁdiz

SPAIN

E-mail address: fernando.leon@uca.es

DEPARTAMENTO DE MATEMÁticas

UNIVERSIDAD DE CÁDIZ

C/ SaCramento 82, 11003-CÁdiz

SPAIN

E-mail address: antonio.piqueras@uca.es 